\title{
MECHANICAL DESIGN FOR ROBUSTNESS OF THE LHC COLLIMATORS
}

\author{
A. Bertarelli, O. Aberle, R.W. Assmann, S. Calatroni, A. Dallocchio, T. Kurtyka, M. Mayer, R. \\ Perret, S. Redaelli, G.Robert-Demolaize,CERN, Geneva, Switzerland.
}

\section{Abstract}

The functional specification of the LHC Collimators requires, for the start-up of the machine and the initial luminosity runs (Phase 1), a collimation system with maximum robustness against abnormal beam operating conditions. The most severe cases to be considered in the mechanical design are the asynchronous beam dump at $7 \mathrm{TeV}$ and the $450 \mathrm{GeV}$ injection error.

To ensure that the collimator jaws survive such accident scenarios, low- $Z$ materials were chosen, driving the design towards Graphite or Carbon reinforced Carbon composites. Furthermore, in-depth thermo-mechanical simulations, both static and dynamic, were necessary.

This paper presents the results of the numerical analyses performed for the $450 \mathrm{GeV}$ accident case, along with the experimental results of the tests conducted on a collimator prototype in CERN TT40 transfer line, impacted by a $450 \mathrm{GeV}$ beam of $3.2 \mathrm{e} 13$ protons, with impact transverse offsets from 1 to $5 \mathrm{~mm}$.

\section{INTRODUCTION}

The design of the LHC collimators must comply with the very demanding specification entailed by the highly energetic beam handled in the LHC.

In nominal conditions the functional specification requires the LHC collimators to absorbe high heat loads with a limited maximum temperature of $50^{\circ} \mathrm{C}$ on the jaws (to limit carbon jaw outgassing in steady-state); moreover a very accurate geometric precision and dimensional stability $(40 \mu \mathrm{m}$ on $1200 \mathrm{~mm})$ are required.

On the other hand, when abnormal beam losses occur, (accident cases), the collimators are expected to withstand without any mechanical failure the induced thermomechanical loads, so as to protect the whole machine from the destructive effects of abnormal beams [1].

To respond to these severe requirements, the mechanical design has been supported from the early phases by in-depth thermo-mechanical analyses, mostly based on the Finite Element Method [2]. Besides, to validate the mechanical design, numerical results of the accident cases have been compared with the outcome of the robustness tests conducted on a collimator prototype in the SPS TT40 transfer line [3].

\section{ACCIDENT CASES}

The two most important accident cases taken into account for the mechanical design are given by [1]:

- Asynchronous beam dump of one module at full energy (9.1e11 protons at $7 \mathrm{TeV}$ over 200ns)

- Injection error at $450 \mathrm{GeV}(3.2 \mathrm{e} 13$ protons over $7.2 \mu \mathrm{s})$
This paper deals with the numerical analysis performed to evaluate the robustness of the LHC secondary collimator (TCSG) in case of injection error; this event provokes the deposition on the collimator jaw of intense heat loads in a very short time that can be essentially regarded as a thermal shock, with a rapid increase of the temperature that causes a fast local deformation and the consequent propagation of stress waves.

\section{FINITE ELEMENT MODEL}

The fast thermal and mechanical phenomena induced in the collimator by the interaction of beam particles with solids are strictly correlated and inter-dependent: it was then chosen to perform a non-linear coupled-field transient analysis to correctly evaluate the temperature distribution, the induced deformations and the stress wave propagation. This analysis was conducted using the ANSYS ${ }^{\circledR}$ Finite Element code.

The input thermal load, in terms of power density distribution, was directly drawn from physical simulation (FLUKA code) [4]; as it is shown in figure 1, the region affected by high energy deposition has a reduced dimension if compared with the one of the whole collimator cross-section. Moreover, considering that the thermal loads do not show an abrupt variation in the longitudinal direction and the large aspect ratio of the collimator bloc $(70 \times 80 \times 1200 \mathrm{~mm})$, it is possible to reasonably assume plane strain behaviour.
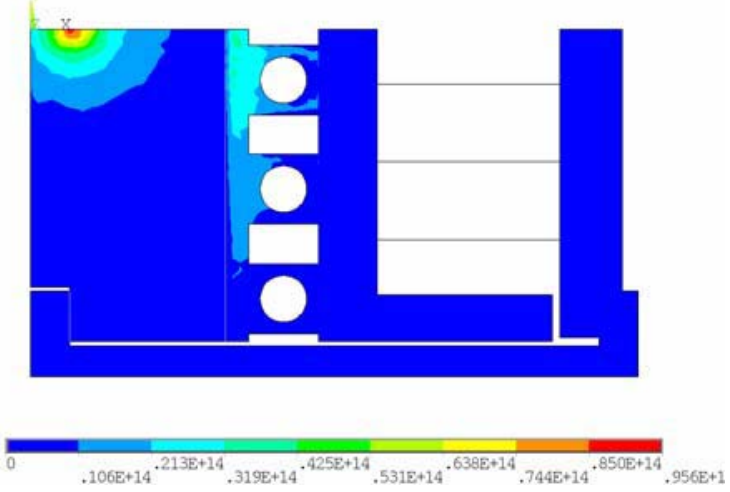

Figure 1: Power density distribution $\left(\mathrm{W} / \mathrm{m}^{3}\right)$ on a crosssection of the secondary collimator bloc in case of injection error $-5 \mathrm{~mm}$ impact transverse offset (halfsymmetry).

In compliance with the plane strain hypothesis, a 2D model of the collimator bloc cross-section was created (figure 1), taking into account both contact interface between the jaw and the heat exchanger (contact elements) and the heat convection on the wet surface of the pipes. 
The thermal conductance at the contact interface was introduced in the FE model as a function of the local contact pressure [5].

Properties of materials implemented in the model, as the coefficient of thermal expansion, the specific heat and the thermal conductivity are temperature dependent. The material adopted for the jaw is a 2-D carbon fibre reinforced carbon composite, chosen for its low-Z number along with good thermal and mechanical properties: in this analysis it is assumed to have a perfectly elastic behaviour since it is expected to remain within its elastic limit.

The thermal shock duration is $7.2 \mu$ s and the evolution of the system was analysed in the first $100 \mu \mathrm{s}$; the integration step was optimised in order to effectively catch the thermo-elastic wave propagation.

An additional analysis was performed over $60 \mathrm{~s}$ to evaluate the thermal evolution of the system far from the impact area and to compare the results with the experimental data retrieved from the temperature probe installed on the collimator for the robustness test.

\section{CALCULATION RESULTS}

\section{Thermal analysis}

During the first $7.2 \mu \mathrm{s}$ the system receives all the energy and reaches the maximum temperature, about $350^{\circ} \mathrm{C}$ on the Carbon/Carbon jaw (figure 2).

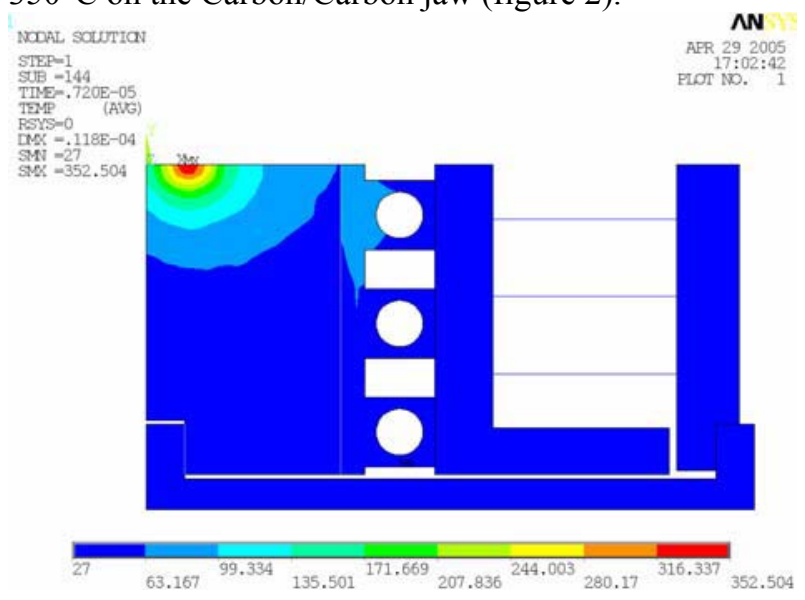

Figure 2: Temperature distribution on 2D collimator cross-section after $7.2 \mu \mathrm{s}(3.2 \mathrm{e} 13$ protons at $450 \mathrm{GeV})$.

This result can be verified with a simple analytical calculation (1), where $\mathrm{P}_{\text {Max }}$ is the maximum power density, $\tau$ is the thermal shock duration, $\rho$ is the Carbon/Carbon composite density, $\mathrm{c}_{\mathrm{p}}$ is the specific heat at $\mathrm{T}_{\mathrm{MAX}}, \mathrm{T}_{\mathrm{REF}}$ is the reference temperature $\left(27^{\circ} \mathrm{C}\right)$ :

$$
T_{M A X}=\frac{P_{M a x} \cdot \tau}{\rho \cdot c_{P}}+T_{R E F}=348^{\circ} \mathrm{C}
$$

Concerning the analysis performed over 60 s, figure 3 shows the evolution of temperature as function of time at the location where the temperature probe has been installed on the collimator jaw for the test.

The numerical analysis gives a maximum temperature variation of $28^{\circ} \mathrm{C}$.

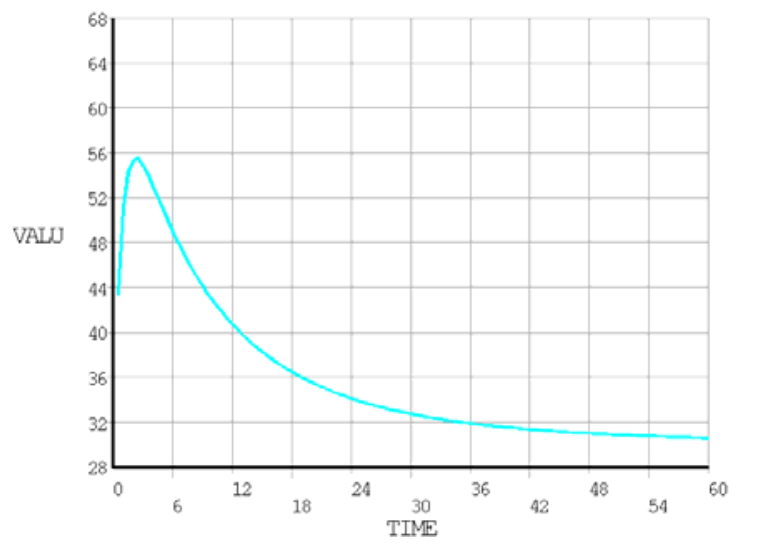

Figure 3: Temperature at probe location as a function of time (60s analysis).

\section{Stress analysis}

The impact transverse offset (i.e. the penetration of the beam into the jaw) used for the simulation is $5 \mathrm{~mm}$; the thermal shock induces a fast local deformation and, as a consequence, the propagation of a stress wave.

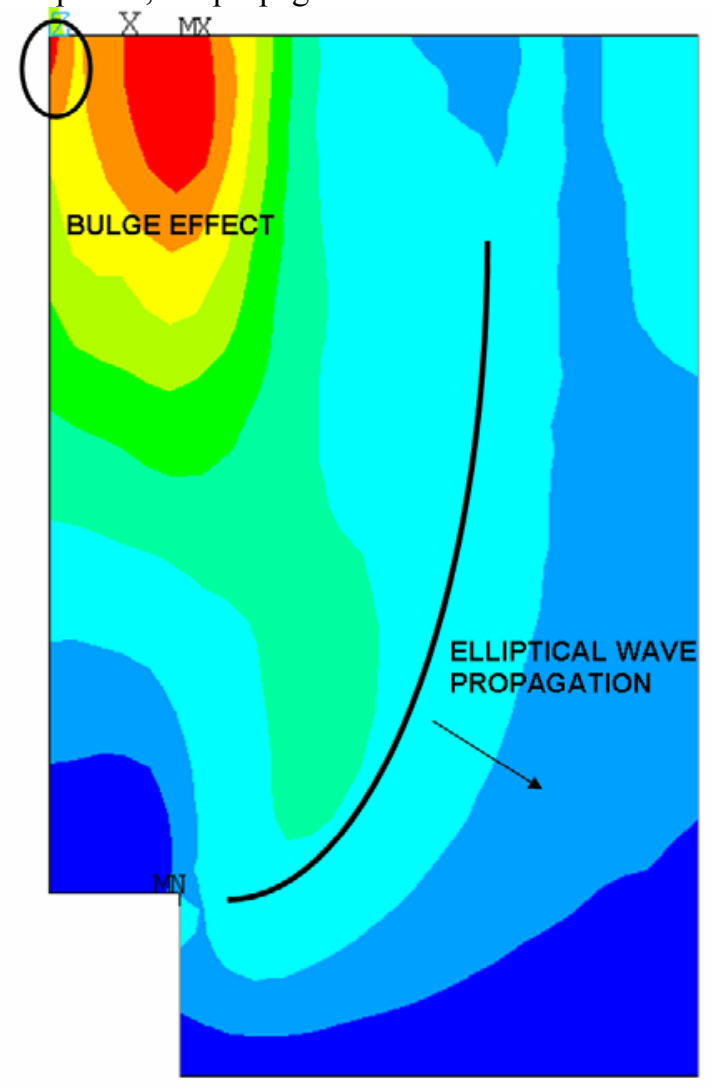

Figure 4: Vertical Stress $\left(\sigma_{\mathrm{y}}\right)$ after $2 \mu \mathrm{s}$ in Carbon/Carbon collimator jaw (half-symmetry).

The 2-D Carbon/Carbon composite used for the collimator jaw is orthotropic (fibres directed as y and $\mathrm{z}$ ); hence the stress waves possess different velocities in $\mathrm{x}$ 
and y direction because of the different Young's modulus in the two directions; as it is shown on figure 4 the wave front takes on an elliptical shape.

A bulge effect due to the reflection of the stress waves on the free surface of collimator jaw can be detected (figure 4); the stress raises here up to $20 \mathrm{MPa}$ (figure 5) but this value should not compromise robustness of the collimator.

More detailed analyses are foreseen to evaluate 3-D dynamic response of the whole collimator structure.

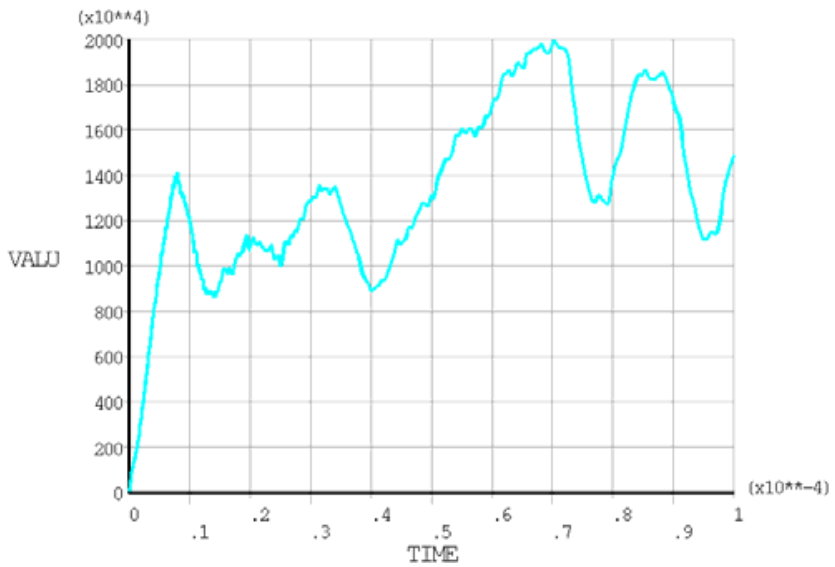

Figure 5: Vertical stress component at $\mathrm{x}=0, \mathrm{y}=0$ (jaw edge) as a function of time (100 $\mu$ s analysis)

\section{ROBUSTNESS TEST}

A robustness test was carried out on a prototype of the secondary collimator in the extraction line of the SPS ring [3]: the goal of this test was to show that an LHC collimator can survive its expected maximum beam load (accident case) without any damage to jaw material, metallic support or cooling circuit.

Each collimator jaw was impacted by five full intensity injection shots $(450 \mathrm{GeV}, 3.2 \mathrm{e} 13$ protons each) with different transverse offsets ( 1 to $5 \mathrm{~mm}$ ), plus many more shots at lower intensity; impact energy was in the range of $2.5 \mathrm{MJ}$, (i.e. approximately equivalent to $0.5 \mathrm{~kg}$ of TNT) [6].

Analyses of the tested components are still ongoing, but after visual inspections no sign of mechanical damage was detected on the collimator jaws [6].

Several temperature sensors were embedded in the collimator jaws: a preliminary analysis of the acquired data shows a temperature increase after a full intensity shot in the range of $20^{\circ} \mathrm{C}$ [7], well in line with the calculations (figure 3). Anyhow, this point must be better investigated as the temperature acquisition rate was too low $(30 \mathrm{sec})$, whereas the measured value might be affected by radiations (all sensors failed after some full intensity shots) [8].

\section{CONCLUSIONS}

In order to ensure the passive protection of the LHC machine aperture in case of abnormal beam losses, the mechanical design of the collimators has been optimised to withstand the thermo-mechanical loads generated in case of accident scenarios. The phenomena induced by the interaction of intense particle beams with solids are complex and highly time-dependent: to asses the effects of the most critical load cases on the collimator components, in-depth calculations were performed during all the design phase; these included non-linear coupledfield transient analyses allowing to evaluate temperature, deformations and stresses on each component as a function of time.

In the case of injection error, calculation results gave maximum stresses below the material admissible limits: these predictions were confirmed by robustness tests performed on a prototype of the secondary collimator in the SPS. After being impacted by several full beams at injection energy, the collimator jaws showed no evidence of mechanical damage. Temperature measurements in the collimator jaws essentially confirmed expected temperature increase.

More extensive analyses are foreseen in the near future to further validate the collimator design.

\section{REFERENCES}

[1] LHC Design Report, Vol. I, Chapter 18, Beam Cleaning and Collimation System, 2004.

[2] A. Bertarelli et al., The Mechanical Design for the LHC Collimators, Proceedings of the $9^{\text {th }}$ European Particle Accelerator Conference (EPAC), p. 545547, Lucerne, 2004

[3] R. W. Assman, LHC Collimation: Design and Results from Prototyping and Beam Tests, These Proceedings, Knoxville, 2005.

[4] V. Vlachoudis et al., Energy Deposition Studies for the Betatron Cleaning Insertion, These Proceedings, Knoxville, 2005.

[5] E. Marotta, S. Mazzucca, J. Norley, Thermal Joint Conductance for Graphite Materials, Electronic Cooling, 8 (2003) 3.

[6] R. W. Assman, Private Communications, CERN, 2005.

[7] G. Robert-Demolaize, S. Redaelli, Private Communications, CERN, 2004.

[8] S. Redaelli et al., Detecting Impacts of Proton Beams on the LHC Collimators With Vibration Measurements, These Proceedings, Knoxville, 2005. 